Instituto Internacional de Investigación y Desarrollo Tecnológico Educativo INDTEC, C.A.

DOI: https://doi.org/10.29394/Scientific.issn.2542-2987.2018.3.8.11.218-233

OAI-PMH: http://www.indteca.com/ojs/index.php/Revista Scientific/oai

\title{
Trastornos del Espectro Autista en Educación Inicial: Experiencia Educativa
}

Autora: Vanessa Alexandra Briceño Matheus Universidad Alonso de Ojeda, UNIOJEDA alexandrab30@hotmail.com

Trujillo, Venezuela

\section{Resumen}

El autismo, al igual que el resto de los trastornos generalizados del desarrollo, están siendo estudiados en la actualidad, no obstante, su causa sigue siendo desconocida. Aunado a esto, el autista es integrado a las escuelas normalistas para mejorar su convivencia y relación con la sociedad. Por ello, los fines de la educación para las personas con Trastornos del Espectro Autista son los mismos que para el resto de los niños(as), con o sin necesidades educativas especiales. Hoy día, el Sistema Educativo Venezolano tiene la necesidad de educar y atender de forma adecuada a todo el alumnado, donde pretenden que en las instituciones educativas no sólo admitan a todos, sino realmente sean escuelas para todos; adaptando el sistema a las personas, en lugar de que sean éstas las que se adapten. El presente artículo tiene como objetivo describir una experiencia educativa de una niña con el trastorno del espectro autista en el ambiente de aprendizaje en el nivel de educación inicial. En cuanto a la parte metodológica, se fundamenta en un estudio de caso con un diseño de campo. Teniendo como resultados, integración con los niños, niñas y docentes, mirada centrada en las personas, permanencia sentada por un tiempo determinado, cumplimiento de instrucciones básicas.

Palabras clave: educación; enseñanza individualizada; educación especial. 


\title{
Asds in Learning Environments of Early Education: Educational Experience
}

\begin{abstract}
Autism, like the rest of the pervasive developmental disorders, are currently being studied; however, their cause remains unknown. In addition, the autistic is integrated into normal schools to improve their coexistence and relationship with society. Therefore, the purposes of education for people with Autism Spectrum Disorders are the same as for the rest of the children, with or without special educational needs. Today, the Venezuelan Educational System has the need to educate and adequately serve all students, where they pretend that in educational institutions not only admit everyone, but really are schools for all; adapting the system to people, instead of being the ones that adapt. This article aims to describe an educational experience of a girl with autism spectrum disorder in the learning environment at the initial education level. Regarding the methodological part, it is based on a case study with a field design. Taking as results, integration with children and teachers, a peoplecentered look, sitting for a certain time, compliance with basic instructions.
\end{abstract}

Keywords: education; individualized instruction; special education. 


\section{Introducción}

Los desórdenes del trastorno del espectro autista, específicamente el autismo, está basado en muchas hipótesis para la mayoría de los investigadores interesados en el tema por muchas razones. Por consiguiente, las personas con Trastornos del Espectro Autista están en su propio mundo como se le dice popularmente, donde tienen sus propias normas, placeres y ocupaciones; De igual forma, están limitados en los aspectos de conciencia, conocimiento tanto de sí mismo como de los demás. Todo ello origina, poca comunicación y establecimiento de lazos sociales lo cual genera aislamiento, falta de comprensión de las emociones de los demás, comportamientos extraños, fijaciones, entre otros.

Desde el punto de vista pedagógico, el autismo se estableció como aquella complejidad que tienen los niños que padecen Trastorno del Espectro Autista, al momento de procesar toda la información que recoge del medio que lo rodea. Cabe destacar, algunos niños autistas presentan nulidad en el habla, sin embargo, lo que presentan el habla tiene ecolalia.

En cuanto a la intervención educativa, la escuela juega un papel importante en el desarrollo cognitivo y social de los autistas, pues tiene el deber de integrar a todos los niños(as) independientemente de sus características personales, para así crear un ambiente propicio para apoyar y dar respuesta a las necesidades de ellos. Así lo establece la Ley Orgánica de Educación, LOE (2009): en cuyo artículo 6 literal c contempla garantizar el acceso al Sistema Educativo a las personas con necesidades educativas o con discapacidad, mediante la creación de condiciones y oportunidades.

Es decir, el Sistema Educativo Venezolano tiene a disposición una educación de calidad y adaptada a las necesidades de cada uno de los niños(as) con diversidad funcional, para dar la adecuada respuesta en materia educativa la cual parte del concepto de inclusión, siendo la atención a la diversidad una necesidad que abarca a todos los niveles educativos. Al 
respecto, Schreibman y Koegel (1981), expresan:

Los niños autistas pueden aprender, pero parece que sólo lo hacen en condiciones de aprendizaje muy cuidadas. No aprenden apenas a menos que se sigan, de forma muy escrupulosa, reglas específicas de enseñanza, identificadas a través de la investigación en el área del aprendizaje. En el tratamiento de las personas autistas, el control adecuado del medio para producir aprendizaje es actualmente el recurso esencial, y bastan pequeñas desviaciones en la conducta del profesor para que se produzcan graves perturbaciones en el aprendizaje del niño autista. (pág. 515).

En general, es beneficioso para los niños(as) con Trastornos del Espectro Autista acudir a las instituciones educativas normalistas puesto que, se benefician de la interacción de los niños sin diversidad funcional, el cual se ha demostrado que las condiciones de integración, pues facilita el aprendizaje y la generalización de las adquisiciones (Riviére, 2001).

Significa entonces, en la escuela hay un ambiente de apoyo como el que ofrecen los propios niños, sin embrago, estos apoyos no siempre se dan de manera espontánea en los compañeros del niño autista, por ende, la labor del maestro es provocarlos para ayudarlos a que vayan saliendo de ellos por sí solos. El presente artículo tiene como objetivo describir una experiencia educativa de una niña con el trastorno del espectro autista en el ambiente de aprendizaje en el nivel de educación inicial en el Centro de Educación Inicial Los Capullitos.

\section{EI Problema}

Resulta oportuno, en el Centro de Educación Inicial Los Capullitos está inscrita por primera vez una niña con el Trastorno del Espectro Autista, de 4 años y 11 meses, presentando características definidas de diversidad funcional a saber una conducta deambulatoria (ir de un sitio a otro sin finalidad), mínimo contacto visual, su lenguaje está formado por monosílabos, 
entre otros. Por ello, el presente artículo hace referencia a la intervención pedagógica de las docentes desde su ingreso a la institución, hasta la actualidad, para evidenciar los progresos en cambios conductuales, emocionales y de lenguaje.

\section{Referentes teóricos}

\subsection{Autismo}

El autismo se caracteriza por la cantidad de restricciones referentes al desarrollo en cuanto al proceso de la imaginación, comportamiento, proceso comunicacional e interacción social recíproca. Al respecto, Miguel y Rodríguez (2009:4), definen el término autismo "proviene del griego, significa sí mismo, fue tomado por Kanne para referirse a la incapacidad para establecer relaciones sociales, a diferencia de Bleuler, quien lo usaba para hacer referencia a las personas con una deficiente imaginación".

Esto significa que, está conceptualizado como un trastorno de origen neurobiológico dando lugar a un desarrollo diferente en las áreas problemáticas como es la comunicación en sus dos vertientes verbal y no verbal, de igual forma, interacciones con el medio que lo rodea y la flexibilidad de la conducta. Por su parte Frith citado por Galindo (2013:4), lo define como "un trastorno del desarrollo que ejerce una influencia duradera en todos los aspectos del desarrollo social, lingüístico y cognitivo del niño. Es un trastorno orgánico del cerebro, posiblemente con un origen genético". Es decir, este trastorno cerebral afecta la capacidad de comunicación e interacción con las personas de su entorno tanto familiar como social, por lo general esta conducta se hace evidente en los primeros tres años de vida.

En cuanto al trastorno autista, Office of Superintendent of Public Instruction, Oficina de la Superintendencia de Enseñanza Pública (2003:7), definen el Autismo como aquellos "niños que se les dificulta la interacción social, comunicación expresiva, receptiva, exhiben patrones de conducta, 
interés, actividades restringidos, repetitivos y estereotipados" Por ende, este trastorno se observa desde el nacimiento hasta los tres años de edad, por medio de las pruebas de retrasos en cuanto a interacción social, habla o juego simbólico.

\subsection{Trastorno del Espectro Autista (TEA)}

Actualmente se consideran los Trastornos del Espectro Autista según la Federación Autismo Andalucía (2005a:8), como "trastornos neuropsiquiátricos que, presentando una amplia variedad de expresiones clínicas, son el resultado de disfunciones multifactoriales del desarrollo del sistema nervioso central". En otras palabras, el autismo constituye un trastorno que se basa en el aspecto de conducta, sin embargo, está ligado a varios trastornos, en las funciones cerebrales biológicamente condicionadas.

Dentro de esta misma línea, la Federación Autismo Andalucía (2005b, pág. 9): exponen entre las características más predominante en los niños autistas son las siguientes:

Alteración del desarrollo de la interacción social recíproca. Se visualizan complicaciones para simpatizar y poner interés con los demás, donde se produce el aislamiento social significativo. Significa, las dificultades que se pueden presentar en el niño y la niña es el entendimiento de los pensamientos, relaciones socioafectivas, creencias y deseos.

Seguidamente, la alteración de la comunicación verbal y no-verbal se evidencia alteraciones en la mirada, pocas acciones gestuales, falta de lenguaje oral. En otras palabras, los niños y niñas con autismo presentan dificultad en comprender la importancia de la comunicación y su aplicación con el entorno que lo rodea, sólo lo emplea cuando necesita o rechaza, presentando ecolalia y estereotipo de él o de los temas de su interés.

De igual forma, repertorio restringido de intereses y comportamientos 
pues, se interesa por realizar conductas como aleteo de manos, balanceos, dar vueltas, entre otras, ayudados de algunos objetos, sin embargo, se le dificulta seguir rutinas diarias. De igual forma, su capacidad de imaginar está ausente, donde presenta dificultad para el juego simbólico. Es decir, tienen problemas para comprender la actividad que se le presenta, así como también, separar, simbolizar y poner en orden su pensamiento, de igual forma, el entender los juegos de roles o imaginativos. Por ende, el docente tiene la capacidad de reforzar estas conductas con instrucciones firmes y simples para llevar acabo la actividad.

\subsection{Integración escolar con los niños autista}

El proceso educativo de los niños con Trastorno del Espectro Autista es un reto complejo para los entes educativos, de manera tal, que se facilite el proceso de inclusión social y educativa de los niños con diversidad funcional, igualmente, facilita el proceso de interacción entre el maestro, alumnos y el niño(a) con TEA, pues se logra que la enseñanza sea un proceso satisfactorio, viable y gratificante para todas las partes tales como docentes, padres y representantes.

En esta intención de dar una respuesta adecuada al continuo de necesidades educativas de niños(as) con TEA en los centros educativos, se hace necesario trabajar de forma cooperativa entre terapistas, directivas de la institución junto a los padres, por ello, Jury y Ballesteros (2009:s.p.), plantean que los profesores:

- Elaboren programas específicos individualizados y adaptados para cada niño, teniendo en cuenta las competencias, estrategias comunicativas utilizadas; las cosas que puede o no realizar con ayuda.

- Realizar el trabajo en grupos pequeños, para facilitar el proceso de aprendizaje del niño. 
- El ambiente de aprendizaje debe ser simple, organizado y bien estructurado, con el propósito de afianzar rutinas cotidianas.

- En el ambiente de aprendizaje se recomienda evitar imágenes distractoras al niño.

- De igual forma, proporcionarle suficiente tiempo y espacio para procesar la información enseñada, al momento de presentar un episodio de frustración, para ello, es necesario alejarlo del sitio y realizar un descanso. En función de esto, al pasar el tiempo el docente puede continuar con la actividad.

- Hacer uso de materiales y estrategias que centren el interés del niño con respecto a la actividad.

- El ambiente de aprendizaje debe estar acorde a la actividad a desarrollar, donde se organizan los materiales para que el niño pueda visualizarlo e identificarlo de manera fácil.

- Hacer uso de los carteles para exponer la rutina a cumplir a fin de facilitar el proceso de enseñanza en cuanto a la información proporcionada.

- Se hace necesario aplicar actividades tanto en colectivo como individual, donde se promueva un objetivo y aprendizaje concretos.

- Con respecto a las tareas asignadas se debe tener en consideración las características particulares del niño(a), con la ayuda del docente.

- Realizar una exploración acerca de los intereses y gustos del niño(a) a fin de facilitar la participación y aprendizaje.

\section{Marco metodológico}

De acuerdo con la problemática planteada en el presente artículo con los objetivos que de él se derivan y las bases teóricas acerca del mismo, la presente investigación es un estudio de caso. Según Stake (2005), citado por Álvarez y San (2012:s.p.). "El estudio de casos es el estudio de la particularidad y de la complejidad de un caso singular, para llegar a comprender su actividad en circunstancias importantes". 
En función de esto, el tipo de diseño es de campo según Sabino (2002:85), el innegable valor reside en que permite a los investigadores, las verdaderas condiciones en las cuales se han conseguido los datos, posibilitando su revisión o modificación, en el caso del surgimiento de dudas respecto a su calidad, lo cual garantiza un mayor nivel de confianza para el conjunto de la información obtenida.

Por otra parte, los métodos de recolección de datos utilizados para obtener la información pertinente fueron la observación directa y el diario del investigador, a fin de recolectar, registrar con el propósito de almacenar toda la información necesaria para la sistematización de la experiencia educativa. En este sentido, al estudiar las diferentes técnicas para el análisis de datos del estudio, se considera la triangulación temporal de tipo tiempo, donde se comprueba la información recabada de los registros y observaciones, antes, durante y después de la intervención pedagógica.

\subsection{Sujeto}

Una niña venezolana que presenta dificultad en el desarrollo tanto psicológico como motor, especialmente en el proceso de socialización de 4 años 11 meses de edad, la cual tiene conductas planteadas por los autores que investigan el síndrome del espectro autista.

\subsection{Aparatos, materiales, escenario}

Se realizó el proceso de observación donde se plasmó en el diario del investigador, las actividades que realizaba con juguetes y asignaciones pedagógicas. Cabe destacar, los registros se llevaron a cabo en el ambiente de aprendizaje del Centro de Educación Inicial Los Capullitos.

\subsection{Procedimiento}

Se realizó el proceso de observación a la niña en situaciones libre y 
dirigidas por el docente en el ambiente de aprendizaje. Este proceso se dio inicio para el año escolar 2014-2015, por medio de acción libre por parte de la niña, luego, de una forma semi controlada, se le proporcionaba diferentes materiales para ser registrada a fin de obtener una respuesta de ella.

\section{Hallazgos}

Para dar cumplimiento con el objetivo describir una experiencia educativa de una niña con el trastorno del espectro autista en el ambiente de aprendizaje en el nivel de educación inicial, se interpretaron las observaciones de la niña junto a otros niños y adultos que interactuaron con ella, durante las observaciones se analizaron los registros descriptivos de la niña, esto significa, sus comportamientos típicos durante la jornada diaria. A continuación, se presenta en el cuadro las acciones que realiza la niña ante diversas situaciones.

Tabla № 1. Características observadas en la niña con el Trastorno del Espectro Autista.

\begin{tabular}{|c|c|c|}
\hline ANTES & DURANTE & DESPUÉS \\
\hline $\begin{array}{l}\text { Se acerca a las personas } \\
\text { para hacer cumplir con } \\
\text { sus deseos por medio de } \\
\text { tomar la mano de la } \\
\text { persona para llevarla al } \\
\text { lugar deseado, sin hacer } \\
\text { contacto visual. }\end{array}$ & $\begin{array}{l}\text { Durante las prácticas } \\
\text { educativas, la niña } \\
\text { comenzó a tener un leve } \\
\text { contacto visual con otras } \\
\text { personas para hacer } \\
\text { cumplir con sus intereses } \\
\text { personales. }\end{array}$ & $\begin{array}{l}\text { Establece contacto visual } \\
\text { para realizar cualquier } \\
\text { actividad pedagógica, } \\
\text { social y comunicacional. }\end{array}$ \\
\hline $\begin{array}{l}\text { Cuando se le proporciona } \\
\text { objetos que son de su } \\
\text { gusto, los agarra sin tener } \\
\text { contacto visual con la } \\
\text { persona, solo ve el objeto. } \\
\text { En cambio, cuando no es } \\
\text { de su gusto solo rechaza. }\end{array}$ & $\begin{array}{l}\text { En este proceso, empezó } \\
\text { a relacionarse con otros } \\
\text { objetos ofrecidos por el } \\
\text { adulto o niño(a) que } \\
\text { convive diariamente con } \\
\text { la niña. }\end{array}$ & $\begin{array}{l}\text { Busca objetos de } \\
\text { diferentes funciones a } \\
\text { saber tacos, dominó, } \\
\text { muñecas, objetos de } \\
\text { cocina, entre otros. }\end{array}$ \\
\hline
\end{tabular}

Fuente: La Autora (2016). 
Tabla N. 1.1. Continuación. Características observadas en la niña con el Trastorno del Espectro Autista.

\begin{tabular}{|c|c|c|}
\hline ANTES & DURANTE & DESPUÉS \\
\hline $\begin{array}{l}\text { Es notorio en su } \\
\text { comportamiento afectivo } \\
\text { tiende a aislarse del } \\
\text { grupo, indiferentemente si } \\
\text { es adulto o no. }\end{array}$ & $\begin{array}{l}\text { Su cambio fue paulatino, } \\
\text { comenzó a integrarse al } \\
\text { grupo de niños y niñas } \\
\text { junto a las docentes en las } \\
\text { diversas actividades } \\
\text { realizadas dentro del } \\
\text { ambiente de } \\
\text { aprendizaje por espacio } \\
\text { de tiempo muy cortos. }\end{array}$ & $\begin{array}{l}\text { Se evidencia el progreso } \\
\text { la niña, pues se integra } \\
\text { inmenso que ha tenido al } \\
\text { grupo por espacio de } 15 \text { a } \\
20 \text { minutos en las } \\
\text { diferentes actividades } \\
\text { planificadas. }\end{array}$ \\
\hline $\begin{array}{l}\text { Cuando desea que la } \\
\text { carguen, agarra las } \\
\text { manos de la persona sin } \\
\text { establecer contacto } \\
\text { visual. }\end{array}$ & $\begin{array}{l}\text { Para este momento, la } \\
\text { niña pronunciaba } \\
\text { palabras donde emitía su } \\
\text { deseo de cargarla } \\
\text { haciendo contacto visual } \\
\text { de manera muy rápida. }\end{array}$ & $\begin{array}{l}\text { Realiza contacto visual y } \\
\text { pronuncia frases para que } \\
\text { la carguen y hacer otras } \\
\text { acciones. }\end{array}$ \\
\hline $\begin{array}{l}\text { No establece diferencia al } \\
\text { momento de estar sola o } \\
\text { acompañada, su } \\
\text { comportamiento es el } \\
\text { mismo. }\end{array}$ & $\begin{array}{l}\text { En función de las rutinas } \\
\text { de ubicarla en el grupo de } \\
\text { niño(a) al momento de } \\
\text { jugar, la niña ya } \\
\text { comenzaba a buscar un } \\
\text { poco de compañía. }\end{array}$ & $\begin{array}{l}\text { En las diversas } \\
\text { actividades pedagógicas } \\
\text { y recreativas, la niña } \\
\text { busca el contacto con } \\
\text { otras personas, sin } \\
\text { distinción alguna. }\end{array}$ \\
\hline $\begin{array}{l}\text { Hace fijaciones por } \\
\text { objetos sonoros y } \\
\text { conocidos por ella que se } \\
\text { encuentra en el hogar. }\end{array}$ & $\begin{array}{l}\text { Se evidenció el interés por } \\
\text { el celular, la música y el } \\
\text { silbato, donde se } \\
\text { incorporaba estos } \\
\text { estímulos para realizar las } \\
\text { actividades. }\end{array}$ & $\begin{array}{l}\text { Ha disminuido las } \\
\text { fijaciones por estos } \\
\text { estímulos, sin embargo, la } \\
\text { música la hace bailar y } \\
\text { sonreír en todo momento. }\end{array}$ \\
\hline
\end{tabular}

Fuente: La Autora (2016).

\subsection{Avances de la niña en el ambiente de aprendizaje}

La niña tuvo una adaptación al ambiente de aprendizaje de manera lenta, lloraba mucho porque manifestaba la presencia de la madre, esto la llevó a tener conducta de aislamiento, todo ello, originó dificultad para integrarse al grupo para la realización de la ronda diaria y cumplimiento con las actividades 
planificadas. No obstante, ha evolucionado positivamente, se muestra contenta, cariñosa, sonriente, el llanto ha disminuido casi en su totalidad, de igual forma, establece contacto visual con niños, niñas y adultos, cuando se le llama para dar instrucciones o recordar normas establecidas en el ambiente de aprendizaje.

Cabe destacar, al principio la niña presentó una conducta deambulatoria (iba de un sitio a otro sin finalidad), con un nivel de atención dispersa, actitud que adopta al parecer cuando está cansada. Sin embargo, esta conducta ha cambiado progresivamente, pues comparte con los otros niños y niñas en la ronda diaria, cantando e imitando los gestos de las canciones que le agradan, de igual forma, cuando se está realizando la actividad del día.

Por su parte, menciona las partes del cuerpo en algunos momentos del día a saber nariz, ojos, boca, pie y mano en los objetos de su agrado como la muñeca del espacio de representar e imitar. También, expresa oralmente su género niño y su nombre, al igual, las indicaciones que se les da en los diferentes períodos de la jornada diaria, cuando tiene sueño, hambre o ganas de ir al baño.

En cuanto al período de la planificación, presenta dificultad para cumplirlo, puesto que, su comunicación es poca fluida, por ende, se le intercambia los espacios. Se observa también que, la niña juega en algunos momentos en compañía de otros niños(as), donde se visualiza su preferencia por los objetos de la cocina, muñecas, objetos de armar, tacos, también, manifiesta sonrisa social, mira a la cara, atención a la voz en situación interactiva, seguimiento de órdenes directas (dame, ven, guarda, los espacios están cerrados, no se salga del salón...), conductas anticipatorias de un nivel muy bajo. Al momento de organizar los espacios, requiere del adulto para practicar las normas de orden y limpieza; sin embargo, se le da la instrucción dos veces y se dispone a colaborar con el arreglo de los mismos.

Por otra parte, presenta hábitos de alimentación, higiene, arreglo 
personal y descanso, aunque requiere de las docentes en algunos momentos para comer, ir al baño, colocarse los zapatos y dormir. Sin embargo, al momento de desayunar lo hace casi en su totalidad sola, en cambio a la hora del almuerzo, requiere la presencia completa de la docente.

Ahora bien, las emisiones verbales espontáneas son muy frecuentes, prácticamente durante toda la jornada diaria, al principio eran monosílabos (mamá, papá, arepa, entre otras), contrariamente a la actualidad, pues expresa con claridad sus ideas tales como quiero hacer pipí, quiero agua, entre otras. En cuanto al lenguaje comprensivo, responde a su nombre y al "no" enfático. Entiende, ejecuta órdenes verbales sencillas en situaciones bien definidas y con apoyo gestual.

En cuanto a la expresión plástica, la niña colorea de forma desordenada sin atender espacio ni formas con los colores de sus preferencias, donde manifiesta los nombres de los colores correctamente, entre los que reconoce se encuentran amarillo, azul, rojo, verde, blanco, carne, morado y negro. Cabe destacar, al momento de expresar su opinión lo hace de manera espontánea, pues se hace notorio cuando realiza garabatos manifiesta su agrado.

Por su parte, la expresión corporal ha sido notorio el cambio que ha tenido la niña, pues participa del acondicionamiento neuromuscular donde es notorio que observa tanto a las docentes como a los niños y niñas para copiar los movimientos (estiramiento de manos, pies, cuello) corre, camina, trota, gatea, tiene equilibrio en la tabla con la ayuda de la docente.

Para la expresión musical, disfruta de escuchar música y de cantar las diferentes canciones que cantan los niños(as) tales como el chicle, comió pan, el manduco, los monitos, entre otras. De igual forma, entonar las letras del Himno del Estado Trujillo, es importante resaltar, mientras que ejecuta acciones canta lo que le agrada, asimismo, comienza por prestar atención cuando se proyecta un video o película; sin embargo, requiere de la docente para lograr dicha acción. Adicionalmente, la niña cuenta los números del 1 al 
21, tiene algunas nociones de día y noche. Asimismo, agrupa los objetos atendiendo a una característica específicamente color.

En función de esto, se produjo una transformación en el cambio de actitud para intervenir pedagógicamente a la niña en tres momentos antes, durante y después del C.E.I. "Los Capullitos", a fin de modificar aquellos aspectos, elementos o factores que sean susceptibles de mejora. Bajo este enfoque, se pude concluir que por medio de esta intervención se logró que tanto la niña como las demás personas se integraron al proceso, con una visión de cooperación, amor, sinceridad y tolerancia. Asimismo, se mejoró la actitud de las docentes y personal de la institución para con los niños de necesidades especiales, donde se integraron en algunas actividades pedagógicas, a fin de comprender las capacidades y habilidades de cada una de las personas sin importar sus dificultades.

\section{Referencias}

Álvarez, C. \& San, J. (2012). La elección del estudio de caso en investigación educativa. Gazeta de Antropología, 28(1), artículo 14, ISSN: 0214-7564. Recuperado de:

http://www.ugr.es/ pwlac/G28 14Carmen AlvarezJoseLuis SanFabian.html

Federación Autismo Andalucía (2005a,b). TEA Trastorno del espectro autista. Guía para su detección precoz. Sevilla, España: Edición Junta de Andalucía. Consejería de Salud.

Galindo, S. (2013). "Trastornos del espectro autista en las aulas de educación infantil De Segovia: Propuesta de Intervención con sistemas Alternativos y aumentativos de la Comunicación". Universidad de Valladolid. Escuela Universitaria de Magisterio de Segovia. Campus María Zambrano. Recuperado de: https://uvadoc.uva.es/bitstream/10324/3151/1/TFG-B.193.pdf 
Jury, K., \& Ballesteros, J. (2009). Inclusión Escolar y Autismo. Qué hacer cuando tenemos un niño con autismo en el salón - algunas recomendaciones para los profesores, (s.p.). Recuperado de: http://inclusionescolaryautismo.blogspot.com/2009/12/que-hacercuando-tenemos-un-nino-con.html

Ley Orgánica de Educación (2009). Gaceta Oficial N. 5.929, Extraordinario del 15 de agosto del 2009. Caracas, Venezuela: La Asamblea Nacional de la República Bolivariana de Venezuela.

Miguel, L., \& Rodríguez, S. (2009). Estudio de caso: Autismo infantil. Wordpress.com. Recuperado de:

https://educacionespecial4.files.wordpress.com/2009/06/autismoinfantil . $\mathrm{pdf}$

Office of Superintendent of Public Instruction (2003). Los aspectos pedagógicos de los trastornos del espectro autista. Olympia, WA: Oficina de la Superintendencia de Enseñanza Pública. Recuperado de: http://www.ite.educacion.es/formacion/materiales/185/cd/material com plementario/m4/Aspectos pedagogicos.pdf

Riviére, A. (2001). Autismo, Orientaciones para la intervención educativa. Madrid: Editorial Trotta.

Sabino, C. (2002). El proceso de investigación. Venezuela: Editorial Panapo. Schreibman, L., \& Koegel, R. (1981). A guideline for planning behavior modification programs for autistic children. En Turner, K., Calhoun, K., \& Adams, H. (Eds.). Handboock of Clinical Behavior Therapy, pp. 500-526. New York: Wiley. 


\section{Vanessa Alexandra Briceño Matheus \\ e-mail: alexandrab30@hotmail.com}

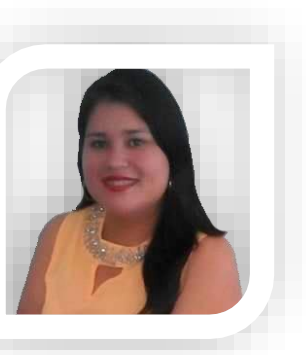

Nacida en Valera, estado Trujillo, Venezuela.

Graduada en la Universidad Nacional Experimental

"Rafael María Baralt". Maestría en Administración de

la Educación Básica Magíster Scientiarum 2014; Licenciada en Educación Mención Básica Integral en

la Universidad de los Andes Núcleo Universitario

Rafael Rangel, 2010. Técnico Superior Universitario en Informática en el Instituto Universitario de Tecnología del estado Trujillo, 2008 y Diplomado en línea Evaluación Neuropsicológica del Desarrollo Infantil, México. Instituto de Investigaciones y Estudios Críticos de Oaxaca A.C., 2016. Actualmente laboro en el Centro de Educación Inicial "Los Capullitos" y en la Universidad Alonso de Ojeda.

El contenido de este manuscrito se difunde bajo una Licencia de Creative Commons Reconocimiento- 